\title{
On Component Reliability and System Reliability for Space Missions
}

\author{
Yuan Chen ${ }^{1}$, Amanda M. Gillespie ${ }^{2}$, Mark W. Monaghan ${ }^{2}$, Michael J. Sampson ${ }^{3}$, Robert F. Hodson ${ }^{1}$ \\ ${ }^{1}$ NASA Langley Research Center, 5 N Dryden St., MS 488, Hampton, VA, 23681 \\ Phone : 757-864-3344, Email: yuan.chen@nasa.gov \\ ${ }^{2}$ SAIC-LX-2, NASA Kennedy Space Center, FL 32899 USA \\ ${ }^{3}$ NASA Goddard Space Flight Center, NASA Goddard Space Flight Center, Greenbelt, MD 20771
}

\begin{abstract}
This paper is to address the basics, the limitations and the relationship between component reliability and system reliability through a study of flight computing architectures and related avionics components for NASA future missions. Component reliability analysis and system reliability analysis need to be evaluated at the same time, and the limitations of each analysis and the relationship between the two analyses need to be understood.
\end{abstract}

Keywords-component reliability; system reliability; space applications;

\section{INTRODUCTION}

A comprehensive components/parts management program and a system reliability and maintainability (R\&M) program are required by NASA for all spaceflight and critical ground support systems to control risk and enhance reliability. The component management program includes components selection, review, verification, approval, traceability, testing, packaging, storage, acquisition, and application [1], while the system R\&M program addresses system R\&M design and operational performance requirements, $R \& M$ engineering analysis and integration, and risk assessment and management [2].

System reliability analysis typically assumes exponential distributions for the components' time to fail. However, depending on workmanship condition, use condition and effectiveness of screening procedures, components may not always operate under the constant failure region, which follows the assumed exponential distribution. Without a comprehensive component management program, it is possible that components may yield early failures or infant mortality or sometimes even wear-out failures under certain use conditions. Therefore, component reliability analysis and system reliability analysis should not be considered separately, but rather be evaluated at the same time, while fully understanding the limitations of each analysis and the relationship between the two analyses is the key.

In this paper, a number of flight computing architectures and related avionics components for launch vehicles are studied, in an attempt to address the fundamental differences between the basics of component reliability and system reliability, and the impact of component reliability on system reliability. In addition, the limitations of system reliability analysis, the misconceptions of either using system reliability to direct component selection or interpreting system reliability in absolute values without fully understanding the assumptions the analysis is based upon, the meaningful relationship of parts reliability and system reliability, and its implementation for space applications which require a high level of reliability of the missions, are also provided in the paper.

\section{SYSTEM RELIABILITY OF COMPUTING ARCHITECTURES}

Various avionics computing architectures similar to existing designs are examined in response to a potential future need to assess and/or design avionics computing architectures for a launch vehicle. Representative computing architectures are selected for detailed study from perspectives of reliability, mass, power, data integrity, software implementation, and hardware and software integration [3]. The six selected architectures are listed below:

a) Fully Cross-Strapped Switched Triplex Voter (FCSSTV)

b) Partially Cross-Strapped Switched Triplex Voter (PCSSTV)

c) Channelized Bussed Triplex Voter (CBTV)

d) Fully Cross-Strapped Switched Self-Checking (FCSSC)

e) Fully Cross-Strapped Bussed Self-Checking (FCSBSC)

f) Channelized Bussed Self-Checking (CBSC)

The selected architectures include both self-checking and voting architectures, with either bussed or switched interconnections, and with various levels of cross-strapping. In order to compare the reliability, mass and power of all the architectures, it is assumed i) all architectures have one-fault tolerant by design, ii) the same lists of sensors and effectors, and iii) the same failure rate and failure criteria for each type of sensor or effector. The sensors and effectors include flight computer (FC), data acquisition unit (DAU), pyro initiation controller (PIC), thrust vector controller (TVC), etc. More details in full paper.

For system reliability analysis, all the selected computing architectures are modeled by Reliability Block Diagram (RBD) Analysis, Cut Set Analysis, and Importance Measure Analysis [4]. The system analyses are based on the mean time to fail (MTTF) of each component assuming an exponential distribution. Fig. 1 shows the reliability plot for the six architectures assuming exponential distributions for all 
components. Table 1 summarizes the architecture reliability at 24 hours and 9 months.

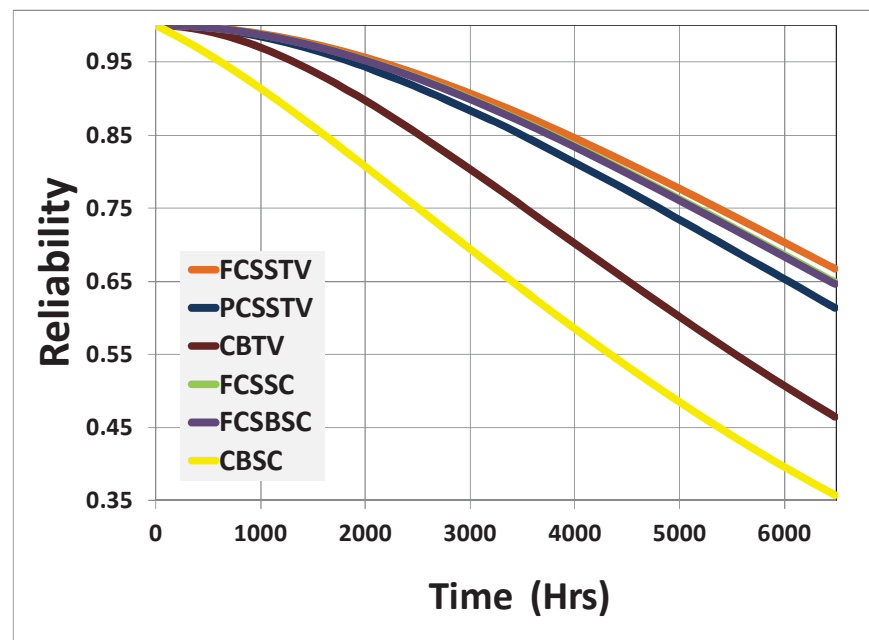

Figure 1. Reliability plot for the architectures assuming exponential distributions for all components.

Table 1. Summary of Architecture Reliability

\begin{tabular}{|c|c|c|}
\hline Architecture & $\mathbf{R}$ (24 hrs) & $\mathbf{R}$ (9 months) \\
\hline FCSSTV & 0.999993 & 0.666999 \\
\hline PCSSTV & 0.999991 & 0.613596 \\
\hline CBTV & 0.999979 & 0.464581 \\
\hline FCSSC & 0.999992 & 0.648547 \\
\hline FCSBSC & 0.999992 & 0.64673 \\
\hline CBSC & 0.998334 & 0.389427 \\
\hline
\end{tabular}

A simple interpretation from Figure 1 and Table 1 may indicate that a lesser stringent component program is needed for short missions, or turning off the avionics system regularly may help improve system reliability. Both concepts fail to consider either the assumptions associated with the system reliability analysis or the assumptions associated with component reliability and, therefore, are mistaken. When proper assumptions are made such as the assumptions described above, system reliability analysis is an excellent approach for comparisons among the architectures and for correlation of the component contribution to the system reliability and therefore leading to the system reliability improvement through component reliability enhancement. However, the assumptions and limitations of the system reliability analysis need to be fully considered so that the results of system reliability are interpreted along with the component reliability to avoid jumping into a misleading conclusion.

\section{COMPONENT RELIABILITY IMPACT ON SYSTEM RELIABILITY}

While system reliability analysis is mainly based on statistics, component reliability analysis relies on statistics as well as technology and physics of failures.

\section{A. Statistics}

The system reliability analysis performed above assumes that component's time to fail follows an exponential distribution. The probability density function of exponential distribution is

$$
f(t)=\lambda \exp (-\lambda t)
$$

where $\lambda$ is the rate parameter. The MTTF of exponential distribution is

$$
M_{\text {TTF }} F_{\text {expential }}=\frac{1}{\lambda}
$$

The probability density function of Weibull distribution is

$$
f(t)=\frac{\beta *(t)^{\beta-1}}{\alpha^{\beta}} \exp \left[-\left(\frac{t}{\alpha}\right)^{\beta}\right]
$$

where $\alpha$ is the scale parameter and $\beta$ is the shape parameter. MTTF of Weibull distribution is

$$
M T T F_{W e i b u l l}=\alpha * \Gamma\left(\frac{1}{\beta}+1\right)
$$

where $\Gamma(\mathrm{z})$ is the gamma function.

Since MTTF is the defining parameter for the component reliability statistics for system reliability analysis assuming exponential distribution, we can translate the exponential distributions used in the above system reliability analysis into a set of Weibull distributions keeping the same MTTF, i.e. $M T T F_{\text {exponential }}=M T T F_{\text {Weibull }}$ to define a set of $\alpha$ and $\beta$ pairs for Weibull using (2) and (4). An example of cumulative density function of the Weibull distributions with $\beta$ ranging from 0.5 and 2 keeping the same MTTF of exponential distribution for a component are plotted in Fig. 2, showing the reliability of the component decreases as $\beta$ decreases for a short mission and increases as $\beta$ decreases for a long mission. This means that the reliability of the component can be anywhere on the curves in Fig. 2 if MTTF remains the same but with different $\beta$ values.

For example, assuming MTTF of the exponential distribution is the same as the MFFT of the weibull distributions with a number of pre-determined $\beta$ values, a set of $\alpha$ and $\beta$ pairs can be calculated through (2) and (4) to define a set of Weibull distributions, all of which will have the same MTTF with other and the same MTTF of the exponential distribution. The cumulative density function of the Weibull distributions with $\beta$ ranging from 0.5 and 2 and the same MTTF for a component are plotted in Figure 2, showing the reliability of the component decreases as $\beta$ decreases for a short mission and increases as $\beta$ decreases for a long mission. This means that the reliability of the component can be anywhere on the curves in Figure 2 if MTTF remains the same but with different $\beta$ values. 


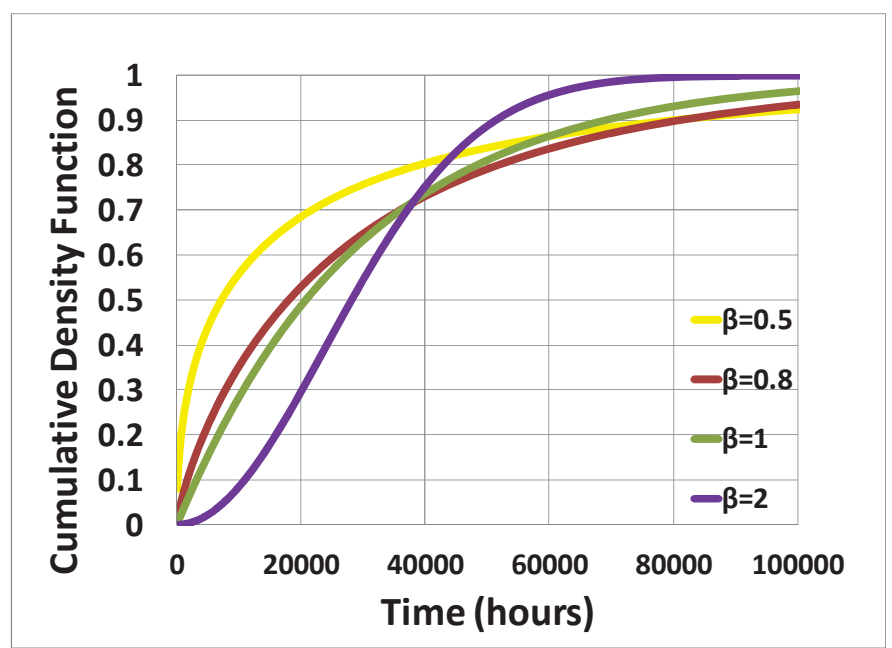

Figure 2. Cumulative density function plot for Weibull distributions with $\beta$ ranging from 0.5 to 2 , keeping the same MTTF of exponential distribution

The contributions of components to the system reliability of the architectures are shown in Fig. 3 [4], from which the flight computers (FC) have the most contribution to the system reliability compared to other components. Using the approach described above to define a set of Weibull distributions while keeping the same MTTF of exponential distributions used for flight computers, the reliability of architecture FCSSTV is recalculated with different $\beta$ values keeping the same MTTF for flight computers only, shown in Fig. 4.

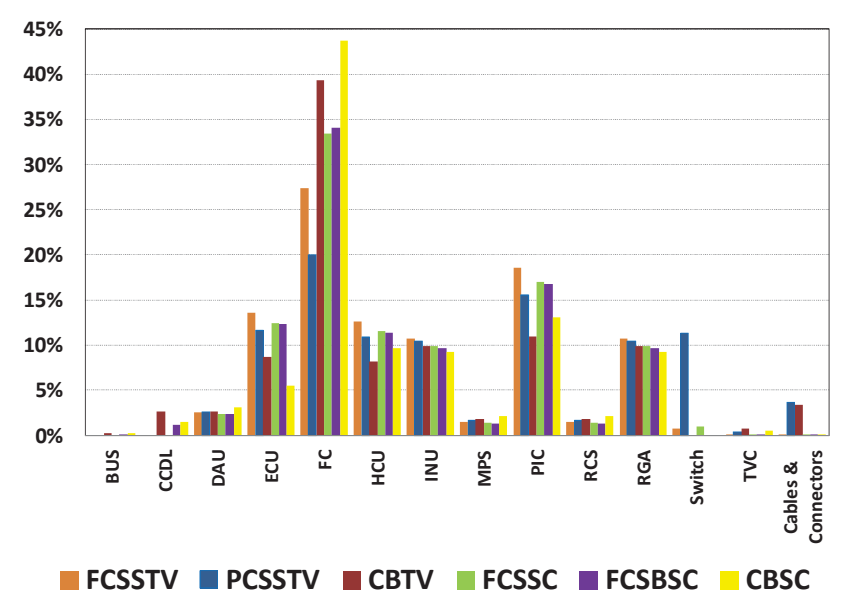

Figure 3 . The percentage of contributions of components to system reliability.

In Fig. 4, only one component, i.e., flight computer, is assumed Weibull distributions with different $\beta$ values while maintaining the same MTTF, there is no changes to other components. The fact that the system reliability numbers are sensitive to the $\beta$ value indicates that the system reliability is a function of the component reliability and, therefore, the workmanship, use condition and effectiveness of screening procedures of the components cannot be overlooked during the system reliability analysis.

Figure 5(a)-(d) show the reliability of all the six architectures with different $\beta$ values while keeping the same
MTTF for flight computers. Fig. 4 and 5 indicate the same trend for all architectures that the change of the shape parameter $\beta$ would yield a different system reliability compared to $\beta$ equal to 1 , which is the common assumption for system reliability analysis.

Due the page limit of the abstract, the more complex impact of multiple components on the system reliability (Relax runs for all components), the different responses of the architectures to the $\beta$ values due to the different contribution distributions of the component, and the cross-over effects of CDF (Relex runs for longer time) and system reliability are to be addressed in the full paper.

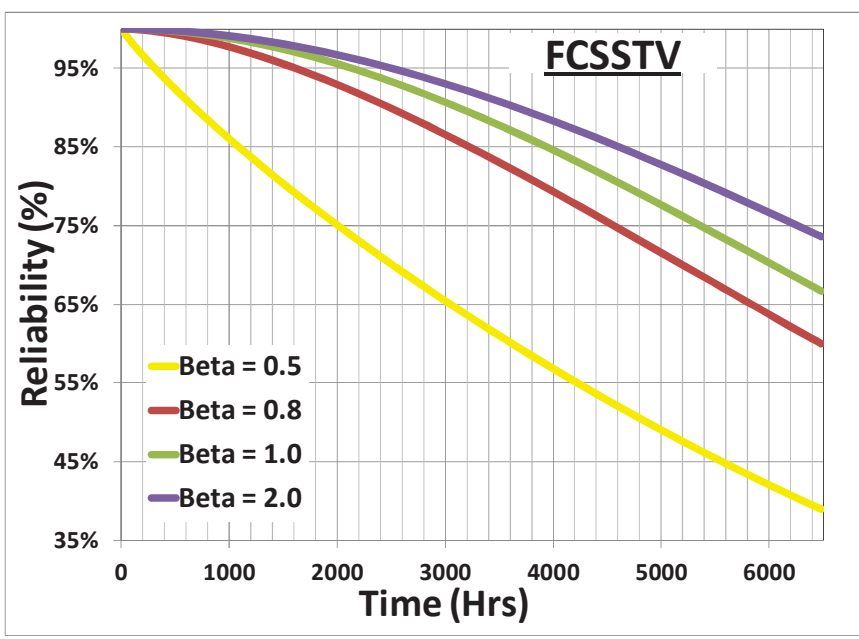

Figure 4. Reliability plot of FCSSTV with $\beta$ ranging from 0.5 to 2 , keeping the same MTTF of exponential distribution for flight computers.

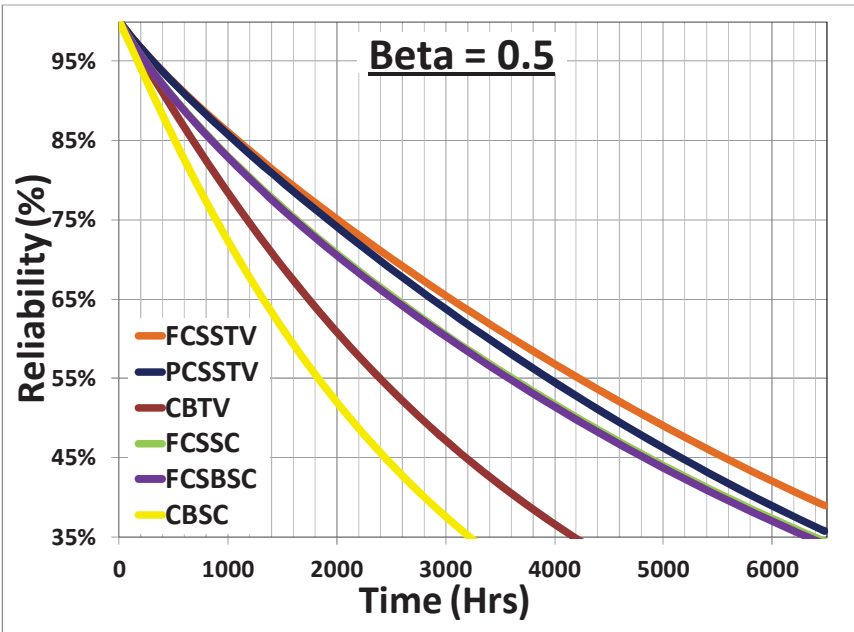

Figure 5(a). Reliability plot of the architectures with $\beta$ equal to 0.5 , keeping the same MTTF for flight computers. 


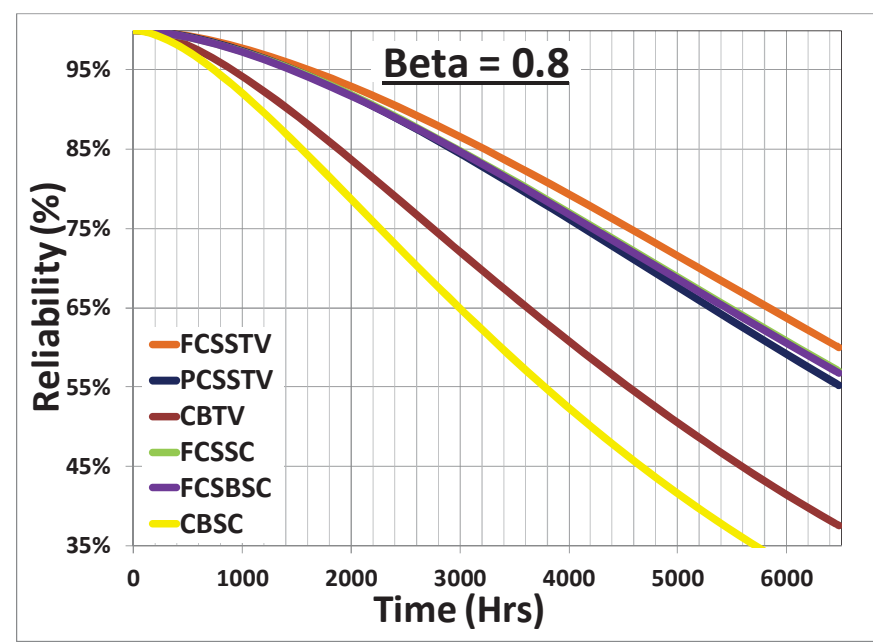

Figure 5(b). Reliability plot of the architectures with $\beta$ equal to 0.8 , keeping the same MTTF for flight computers.

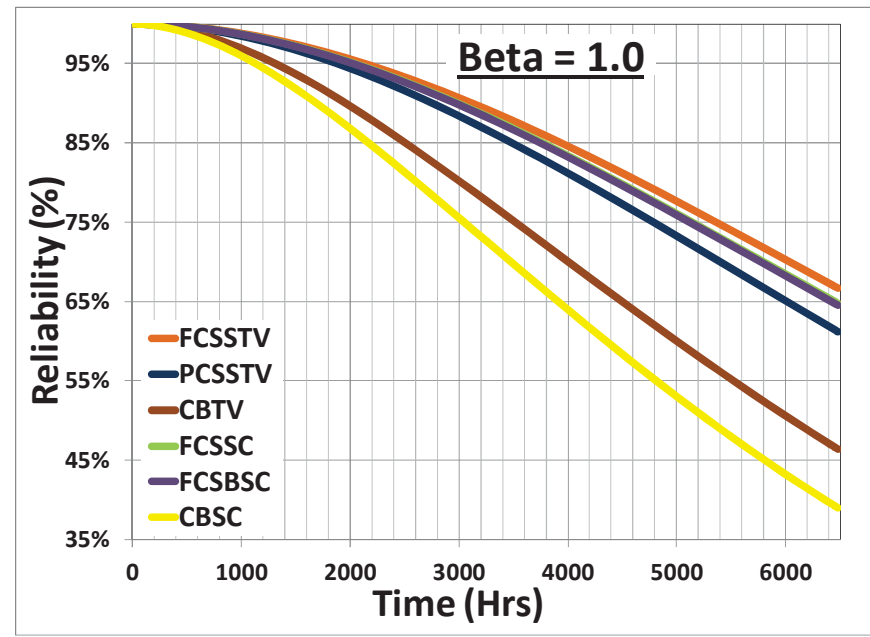

Figure 5(c). Reliability plot of the architectures with $\beta$ equal to 1.0, keeping the same MTTF for flight computers.

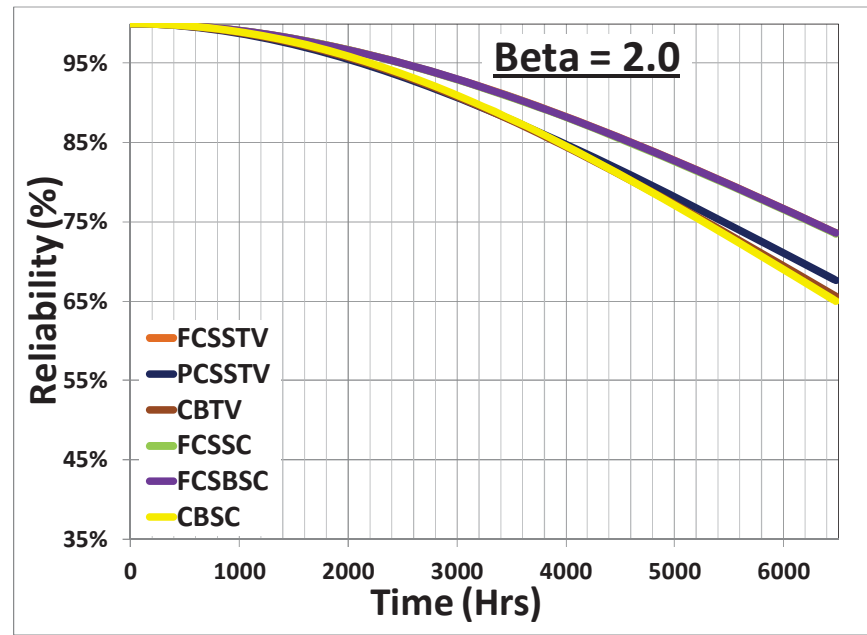

Figure 5(d). Reliability plot of the architectures with $\beta$ equal to 2.0, keeping the same MTTF for flight computers.

\section{B. Failure Modes}

Since the shape parameter $\beta$ corresponds to the different failure modes for components, i.e., infant mortality when $\beta$ is less than 1 , random defects when $\beta$ is equal to 1 , and wear-out when $\beta$ is greater than 1 , the results of system reliability analysis can be misleading if components are not properly upscreened or used under a certain bias condition where different failure modes may occur.

Table 2 gives the system reliability of the architectures at 24 hours and 9 months with $\beta$ ranging from 0.5 to 2 . The architectures do have a different level of sensitivity to the change of $\beta$. While the changes of the system reliability numbers are more evident for longer time, e.g. 9 months, the channelized architectures CBTV and CBSC are more sensitive to $\beta$ values compared to other architectures, since the number of "9"s changes even when $\beta$ changes from 1 to 0.8 . This indicates that the up-screening of the component to ensure that the early failures are excluded is critical to mission success, even for a short mission. It is therefore a critical decision for the mission to evaluate the risk and risk mitigation of using the components with lesser grade which has less stringent upscreening procedures. Cost associated with the use of the components with higher grade is of importance as well; however, different cost models are available, with one example of NASA cost model referenced in [5].

Table 2. Summary of Architecture Reliability with $\beta$ between 0.5 and 2

\begin{tabular}{|c|c|c|c|c|c|c|}
\hline \multirow{2}{*}{ Beta } & \multicolumn{7}{|c|}{$\mathbf{R}(24$ hrs) } \\
\cline { 2 - 7 } & FCSSTV & PCSSTV & CBTV & FCSSC & FCSBSC & CBSC \\
\hline 0.5 & 0.995388 & 0.995412 & 0.994807 & 0.993915 & 0.993867 & 0.992985 \\
\hline 0.8 & 0.999935 & 0.999932 & 0.999878 & 0.999910 & 0.999923 & 0.999825 \\
\hline 1.0 & 0.999982 & 0.999982 & 0.999968 & 0.999976 & 0.999989 & 0.999960 \\
\hline 2.0 & 0.999986 & 0.999984 & 0.999985 & 0.999981 & 0.999994 & 0.999981 \\
\hline \multirow{2}{*}{ Beta } & R (9 months) \\
\cline { 2 - 8 } & FCSSTV & PCSSTV & CBTV & FCSSC & FCSBSC & CBSC \\
\hline 0.5 & 0.390061 & 0.358168 & 0.195661 & 0.344148 & 0.341046 & 0.124876 \\
\hline 0.8 & 0.600525 & 0.551993 & 0.375597 & 0.570492 & 0.567588 & 0.289916 \\
\hline 1.0 & 0.666420 & 0.612354 & 0.463806 & 0.647530 & 0.645730 & 0.389427 \\
\hline 2.0 & 0.736666 & 0.676404 & 0.656380 & 0.734770 & 0.736430 & 0.650573 \\
\hline
\end{tabular}

Majority of the failure or degradation mechanisms are accumulative; some are more aggravated at higher temperature, some are more sensitive to thermal cycling, and some are more prominent under bias or power-on condition. Not all the failure modes are alike and more details to be included for system reliability improvement in the full paper.

\section{Long Missions}

It is evident that the system reliability decreases for long missions. This may not be an issue for short missions such as launch vehicles, but is critical for long mission such as crewvehicles. The statistics assumptions and component assumption described so far enable the architecture reliability comparisons and reliability improvements; however, more studies are needed if the absolute system reliability numbers are used for design/mission decisions. More in the full paper. 


\section{System Reliability Improvement}

System reliability can be improved by enhancing component reliability, adding component and/or module level redundancy, and keeping simple core critical avionics architecture designs. System reliability analysis provides distributions of the failure probability contribution from each component and indicates different reliability improvement path for the architectures. The level of redundancy needed depends on the component reliability, architectures design, mission lifetime, etc. which will be discussed in the full paper.

\section{SUMMARY}

This paper is to address the basics, the limitations and the relationship between component reliability and system reliability through a study of flight computing architectures and related avionics components to show that component reliability analysis and system reliability analysis need to be evaluated at the same time, and the limitations of each analysis and the relationship between the two analyses need to be understood.

\section{REFERENCES}

[1] NPD 8730.2C, "NASA Parts Policy", NASA Policy Directive, 2008.

[2] NPD 8720.1C, "NASA Reliability and Maintainability Program Policy", NASA Policy Directive, April 2008.

[3] R. Hodson, et al, "Heavy Lift Vehicle (HLV) Avionics Flight Computing Architecture Study", NASA Technical Report, 2011.

[4] A. M. Gillespie, M. W. Monahan, Y. Chen, "Comparison Modeling of System Reliability for Future NASA Projects", Reliability and Maintainability Symposium, 2012.

[5] M. J. Sampson, "Cost/Benefit of using COTS EEE Parts in Space", Commercialization of Military and Space Electronics Conference, February, 2003. 